\title{
Evaluating parameters for ligand-based modeling with random forest on sparse data sets
}

\author{
Alexander Kensert ${ }^{1^{*}} \mathbb{( D}$, Jonathan Alvarsson ${ }^{1}$, Ulf Norinder ${ }^{2,3}$ and Ola Spjuth ${ }^{1}$
}

\begin{abstract}
Ligand-based predictive modeling is widely used to generate predictive models aiding decision making in e.g. drug discovery projects. With growing data sets and requirements on low modeling time comes the necessity to analyze data sets efficiently to support rapid and robust modeling. In this study we analyzed four data sets and studied the efficiency of machine learning methods on sparse data structures, utilizing Morgan fingerprints of different radii and hash sizes, and compared with molecular signatures descriptor of different height. We specifically evaluated the effect these parameters had on modeling time, predictive performance, and memory requirements using two implementations of random forest; Scikit-learn as well as FEST. We also compared with a support vector machine implementation. Our results showed that unhashed fingerprints yield significantly better accuracy than hashed fingerprints ( $p \leq 0.05)$, with no pronounced deterioration in modeling time and memory usage. Furthermore, the fast execution and low memory usage of the FEST algorithm suggest that it is a good alternative for large, high dimensional sparse data. Both support vector machines and random forest performed equally well but results indicate that the support vector machine was better at using the extra information from larger values of the Morgan fingerprint's radius.
\end{abstract}

Keywords: Random forest, Support vector machines, Sparse representation, Fingerprint, Machine learning

\section{Background}

Ligand-based modelling is a widely used method where the ligand's activity for a biological target, usually a measurement obtained from a bioassay, can be correlated to certain features of the ligand. The derived model can then be used for predicting the biological activity of new novel chemical compounds. Examples of applications include studies on bio-availability [1], bioactivity of GPCR-associated ligands [2], mitochondrial toxicity [3], organ toxicity [4], hepatotoxicity [5] and aquatic toxicity [6].

In quantitative structure-activity relationships (QSAR), chemical structures are represented as numerical features via algorithms referred to as molecular descriptors. An important example is ECFP (Extended-Connectivity Fingerprints), which are molecular descriptors specifically

\footnotetext{
*Correspondence: alexander.kensert@gmail.com

${ }^{1}$ Department of Pharmaceutical Biosciences, Uppsala University, Uppsala, Sweden

Full list of author information is available at the end of the article
}

developed for structure-activity modelling. The original article of ECFP illustrate the strengths of the algorithm and how it can be applied in a variety of computational chemistry domains [7]. In classification problems ECFP has, for example, been valuable for predicting inhibition of Cytochrome P450, 2D6 and 3A4 [8] and of Escherichia coli dihydrofolate reductase [9]. It has also been applied in quantitative structure-property relationships (QSPR) for studying melting points and aqueous solubility of organic compounds [10].

Machine learning (ML) algorithms is an important component in structure-activity modelling and analysis of compounds, and an example of a widely used method is support vector machines (SVMs). This method has proven to be successful for correlating molecular structures to toxicity and activity of compounds $[3,4,11$, 12]. SVMs have also shown to be useful for drug transport predictions [13] and to model and study interactions of antibiotic compounds [14]. Another important and successful machine learning method is the random

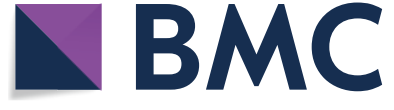

(c) The Author(s) 2018. This article is distributed under the terms of the Creative Commons Attribution 4.0 International License (http://creativecommons.org/licenses/by/4.0/), which permits unrestricted use, distribution, and reproduction in any medium, provided you give appropriate credit to the original author(s) and the source, provide a link to the Creative Commons license, and indicate if changes were made. The Creative Commons Public Domain Dedication waiver (http://creativecommons.org/ publicdomain/zero/1.0/) applies to the data made available in this article, unless otherwise stated. 
forest (RF) [15], which like SVM has proven to be valuable in toxicity and bio-activity studies $[2,5,6,16,17]$, and also for investigating diverse representations of clinical events [18] as well as predicting adverse drug events from electronic health records [19]. RF has been one of the most widely used ML algorithms together with SVM, and studies have demonstrated that RF is a powerful yet easy-to-implement method in both QSAR and descriptor selection [20, 21].

Although extensive and rigorous research have been performed in the area of QSAR and ML methods, there are few comprehensive studies on RF together with Morgan fingerprints [22], a powerful ECFP variant [7], so we set out to study random forest in general, and the random forest implementation FEST (Fast Ensembles of Sparse Trees) in particular, together with Morgan fingerprints.

The Python package Scikit-learn contains a well known and much used random forest implementation which we decided to use as a reference point. Since SVM is highly used in QSAR and since SVM together with molecular signatures has been shown to perform well for this kind of QSAR tasks [23, 24] we decided to include an SVM as well in the study as another reference point.

In this article the following thoughts and ideas are examined further: Perhaps working with hashed fingerprints of smaller sizes would speed up the modeling or make it require less memory without resulting in worse models? How many collisions would hashing to different fingerprint size give rise to in typical QSAR data sets? What effect does different values for the random forest parameter Max features have on the prediction models? What radii should be used for the Morgan fingerprints? Higher values would mean more data and it would seem reasonable to expect more data to give better models but at the cost of modeling time. Would there be a tradeoff there? Random forests do not require the extended parameter search that support vector machines require so can they be trained faster for the QSAR problem?

\section{Methods \\ Data}

Four different public datasets were used in this study, containing 5000-7000 compounds each (Table 1). Three of them were obtained from the tox 21 challenge (https ://tripod.nih.gov/tox21/challenge/data.jsp), and contains data from QHTS assays to identify small molecules that: (1) activate the aryl hydrocarbon receptor (nr-ahr), (2) act as agonists of the estrogen receptor alpha signaling pathway using the BG1 cell line (nr-er), and (3) disrupt the mitochondrial membrane (sr-mmp) [25]. The fourth data set was obtained from the paper "Benchmark Data Set for in Silico Prediction of Ames Mutagenicity" by Hansen and co-workers [26].
Table 1 The data sets used in the study

\begin{tabular}{llll}
\hline Dataset & Negatives & Positives & Sum \\
\hline sr-mmp & 4763 & 884 & 5647 \\
nr-ahr & 5599 & 700 & 6299 \\
nr-er & 5235 & 623 & 5858 \\
cas-N6512 & 3007 & 3502 & 6509 \\
\hline
\end{tabular}

Structure standardization was performed using the IMI eTOX project standardizer (version 0.1.7. https ://pypi.python.org/pypi/standardiser) in combination with the MolVS standardizer (version 0.0.9. https:// pypi.python.org/pypi/MolVS) for tautomer standardization. The Python libraries Matplotlib (version 2.1.0) and Seaborn (version 0.8.0) were used to illustrate the results of this study $[27,28]$.

\section{Morgan fingerprints}

There are numerous ways of generating molecular fingerprints. In this study, the open-source Python framework RDKit (version 2017.09.1) was used to generate Morgan fingerprints [29]. The atomic invariants of these fingerprints use connectivity information similar to the the Extended Connectivity Fingerprints (ECFP) family of fingerprints $[7,29]$. The Morgan algorithm initially assigns an integer identifier to each non-hydrogen atom, then iteratively, by extending the connectivity of each atom to its neighbouring atoms, updates the numerical identifiers based on these neighbouring atoms [7]. There are mainly two parameters to be set for the generation of the Morgan fingerprint: (1) bit size (or hash size) - the length of the bit string for the molecular features to be contained in; (2) radius - the number of neighbours $\times$ bond lengths away to take into account when calculating the identifiers of the atoms. A fingerprint collision occur when a feature falls into a bin (a dataset column) of another feature-resulting in more than one molecular substructure being compressed into a single, now hashed, feature. It is also possible to use an unhashed version of the fingerprint, which means that the compression of the bit string is bypassed and hence encode explicitly defined patterns. In this study, both the unhashed and hashed Morgan fingerprints were generated and compared (Table 2).

\section{Molecular signatures}

In addition to the Morgan fingerprints, molecular signatures [30] were generated and evaluated. The molecular signatures is a molecular descriptor similar to the Morgan fingerprints in the sense that its identifiers are based on the neighbouring of atoms. Contrary to the Morgan fingerprint, the signature descriptors do not hash the information into an index, but generate explicitly defined 
Table 2 Overview of the molecular descriptors used in the study

\begin{tabular}{|c|c|c|}
\hline \multicolumn{2}{|c|}{ Morgan fingerprints } & \multirow{2}{*}{$\begin{array}{l}\text { Molecular signatures } \\
\text { Height }\end{array}$} \\
\hline Hash size & Radius & \\
\hline 128 & 1 & $1-1$ \\
\hline 256 & 2 & $1-2$ \\
\hline 512 & 3 & $1-3$ \\
\hline \multicolumn{3}{|l|}{1024} \\
\hline \multicolumn{3}{|l|}{2048} \\
\hline \multicolumn{3}{|l|}{4096} \\
\hline Unhashed & & \\
\hline
\end{tabular}

Table 3 Overview of the different machine learning methods and parameter settings used in the study

\begin{tabular}{lrlll}
\hline FEST and Scikit RF & & & \multicolumn{2}{l}{ Scikit SVM } \\
\cline { 5 - 5 } Max features $^{\mathbf{a}}$ & Trees & & $\boldsymbol{C}$ & $\boldsymbol{\gamma}$ \\
\hline 0.1 & 10 & & 0.01 & $1 \times 10^{-6}$ \\
0.3 & 30 & & 0.1 & $3 \times 10^{-6}$ \\
1.0 & 100 & 1 & $1 \times 10^{-5}$ \\
3.0 & 300 & 10 & $3 \times 10^{-5}$ \\
10.0 & 1000 & 100 & $1 \times 10^{-4}$ \\
& & 1000 & $3 \times 10^{-4}$ \\
& & $1 \times 10^{4}$ & 0.001 \\
& & $1 \times 10^{5}$ & 0.003 \\
& & $1 \times 10^{6}$ & 0.01 \\
& & $1 \times 10^{\prime}$ & 0.03 \\
& & $1 \times 10^{8}$ & 0.1 \\
\hline
\end{tabular}

aValues indicate the multiplicating factor by the square root of the number of features

substructures, which are then mapped to numerical identifiers. Equivalent to the radius of Morgan fingerprints, signatures have a height parameter which extends away one or several bonds from the investigated atom (Table 2).

\section{Random forests}

Random forests are ensembles of decision trees [31]. According to the strong law of large numbers, growing a large number of decision trees lead to better generalization and prevention of over-fitting. This generalization error converges as the number of trees grow and depend on the strength of and correlation between the individual trees [15, 32]. Importantly, the correlation between the individual trees is reduced by several random processes in the random forest algorithm. First, for the $S$ number of trees in the forest, $S$ number of new datasets are randomly sampled (with replacement). Second, a random subspace method is used which selects a subset of $m$ features from the total number of features $M$ before each split-normally determined by the information gain or Gini impurity metric [33]. The splitting, or branching, continues until it reaches a leaf node, which contains a class label probability. Hence the ensemble of trees produces $S$ outputs for an input $x$, each with its own probability for the classes. This is then averaged across the ensemble of trees to generate a final prediction of the class with the highest probability.

Fast ensembles of sparse trees (FEST) is a software written in $\mathrm{C}$ for learning various types of decision tree committees from high dimensional sparse data [34] that efficiently handles sparse data structures. The current implementation allows for setting different hyperparameter values for RF, such as (1) Max features (the maximum number of allowed splitting features to be considered), (2) number of trees, (3) maximum depth of the tree, and (4) relative weight for the negative class.

Scikit-learn's random forest classifier (Scikit RF) is part of an open source machine learning framework in Python [35]. Scikit RF allows for experimenting with the same hyperparameters as the FEST implementation and many more. Like FEST, Scikit RF supports sparse matrix operations, but also parallelization, which can speed up model training when using multiple CPUs.

In this study the information gain metric was used for both RF implementations, and Maximum Depth parameter was set to large enough (1000) to not affect the tree depth. To accommodate for inbalanced datasets, the parameter relative negative weights (FEST) and class weights (Scikit RF) were set to balance the classes. Max features and Number of trees were two hyperparameters selected for investigations (Table 3).

\section{Support vector machines}

Scikit-learn's C-support vector classifier (Scikit SVM) [35] is based on the Libsvm implementation of support vector machines [36]. Like the FEST and Scikit RF implementations, Scikit SVM handles sparse data structures in an efficient way. Support vector machines use kernel functions to non-linearly map inseparable inputs $X$ to a higher dimensional space $\phi(X)$ where they can be linearly separated by a hyper-plane. Scikit SVM handles high dimensional data efficiently by solving the dual problem, where instead of learning a weight vector $w$ of possibly thousands of dimensions which require significant computational power, instead learns a vector $\alpha$ in the dual problem which contains all zeros except for the support vectors. In this study, the radial basis function (RBF) was used as kernel, and the hyperparameters investigated 
Table 4 Number of of compounds and number of descriptors generated for the different data sets and molecular descriptors

\begin{tabular}{|c|c|c|c|c|}
\hline & \multicolumn{4}{|l|}{ Data sets } \\
\hline & sr-mmp & nr-ahr & nr-er & cas $\mathrm{N} 6512$ \\
\hline \multicolumn{5}{|c|}{ Morgan fingerprints } \\
\hline \#Compounds & 6299 & 5647 & 5858 & 6509 \\
\hline Radius 1 & 3352 & 3525 & 3350 & 2935 \\
\hline Radius 2 & 21,542 & 23,695 & 21,974 & 19,131 \\
\hline Radius 3 & 49,764 & 55,725 & 51,200 & 48,325 \\
\hline \multicolumn{5}{|c|}{ Molecular signatures } \\
\hline \#Compounds ${ }^{\mathrm{a}}$ & 6193 & 5546 & 5761 & 6396 \\
\hline Height 1-1 & 504 & 514 & 487 & 405 \\
\hline Height 1-2 & 7524 & 8021 & 7547 & 6758 \\
\hline Height 1-3 & 33,237 & 36,601 & 33,900 & 31,581 \\
\hline
\end{tabular}

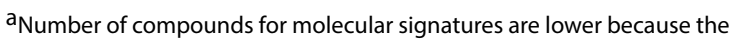
algorithm couldn't generate descriptors for some compounds

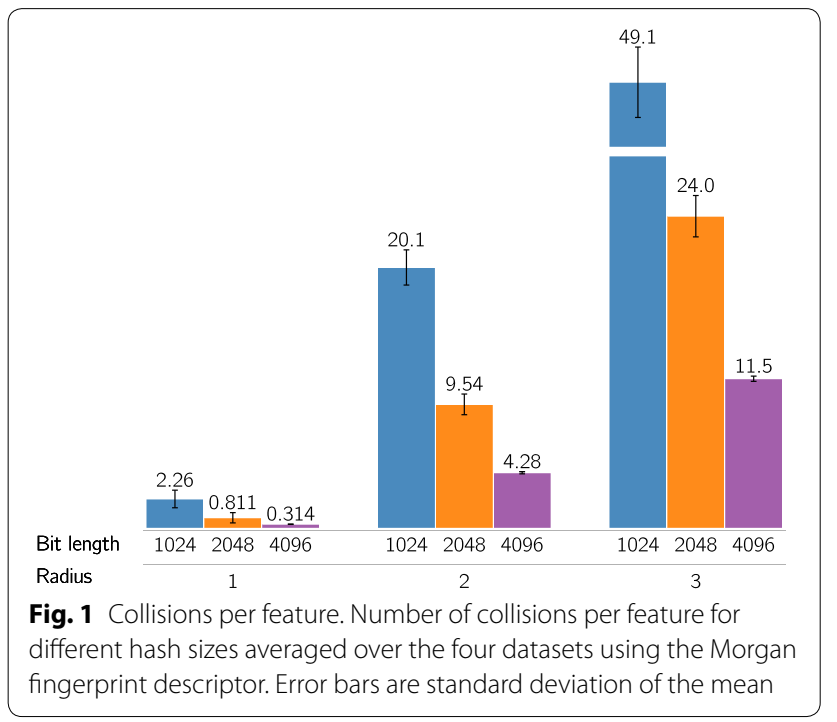

were Cost $(C)$ and the kernel parameter gamma $(\gamma)$ (Table 3).

\section{Model evaluation}

In this investigation, ROC-AUC was used as metric for evaluating the different models. A receiver operating characteristic curve (ROC-curve) is a graphical plot illustrating the true positive rates (TPR) against the false positive rate (FPR) at different thresholds. The AUC is then the area under this curve, which has shown to be a valid and advantageous metric for evaluating ML algorithms [37]. For each hyperparameter combination, a 5-fold randomly shuffled cross-validation was utilized to yield a cross-validated ROC-AUC score; the procedure was repeated five times to produce a more robust metric with respect to the models performance. In the case of Scikit SVM, ROC-AUC scores were calculated with respect to the decision function.

\section{Results and discussion}

We studied the effect of two random forest implementations (Scikit RF and FEST) and a C-support vector classifier (Scikit SVM) on sparse datasets for ligand-based modelling. Specifically evaluating combinations of parameters according to Tables 2 and 3, and their effect on ROC-AUC, memory usage and run-time.

All datasets were subjected to descriptor generation using Morgan fingerprints and molecular signatures (Tables 2 and 4). Hashed versions of the Morgan fingerprints were generated with $128,256,512,1024,2048$, and 4096 bins.

Every hyperparameter combination of each ML method was trained on the datasets with every descriptor parameter combination (Tables 1, 2 and 3). ROC-AUC, memory usage and run-time were measured for all runs.

Overall the tested machine learning algorithms and descriptors produce models of similar prediction capacity (Fig. 5) which is not surprising considering these are all commonly used methods in QSAR and should be expected to produce good results. There are however some differences that might be relevant depending on use cases.

\section{Effect of hashed versus non-hashed features}

To illustrate both the way hashing reduces the dimensions of a data set, as well as decreases the "resolution" of the fingerprints, collisions for all data sets using the Morgan fingerprint were plotted (Fig. 1).

Further investigation into hash sizes illustrated that there are no obvious differences between Scikit SVM, Scikit RF and FEST in terms of ROC-AUC scores, with a plateauing of performance beyond $1024 \mathrm{bit}$, and a spike from 4096 to unhashed (Fig. 2; "Appendix"). However, our results show that unhashed fingerprints yield better performance compared to hashed fingerprints according to the difference between the areas under the two ROC curves using the method of Hanley and McNeil [38] (for the full result table, see Additional file 1: Table S1). For all statistically significant cases $(p \leq 0.05)$ were the ROC curve area for unhashed fingerprints larger than for hashed fingerprints for each dataset, respectively. Also, for almost all of the non-significant cases (265 out of 268 cases) were the ROC curve area larger for unhashed fingerprints than for hashed fingerprints. Considering the significant improvements in predictive performance, with no pronounced difference in memory usage or runtime (Figs. 3, 4), the preferred choice should be to use 


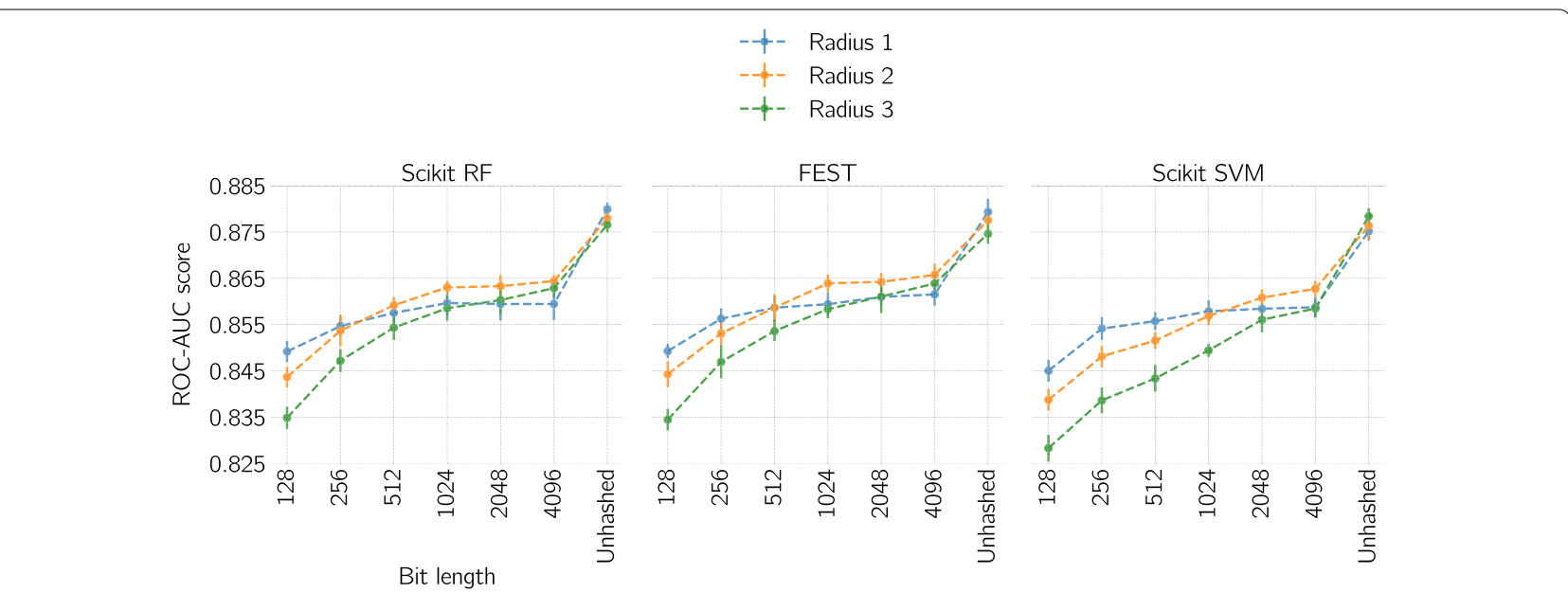

Fig. 2 Effect of hash size and radius on predictive performance with Scikit RF, FEST and Scikit SVM. Each data point is an average of the best ROC-AUC score for each dataset. Error bars are pooled standard deviations

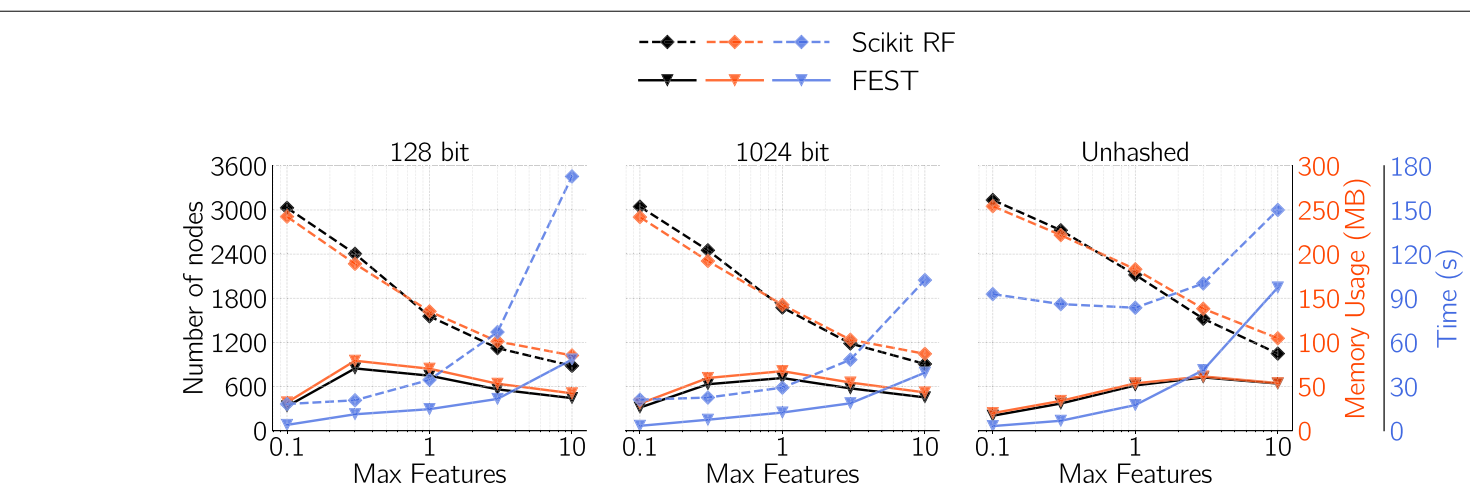

Fig. 3 Effect of Max features and hash size on number of nodes (per tree), memory usage and run time for the two random forest implementations with 1000 trees. Data points are average values of the four datasets, and although imperceptible due to minuscule values: error bars are pooled standard deviations
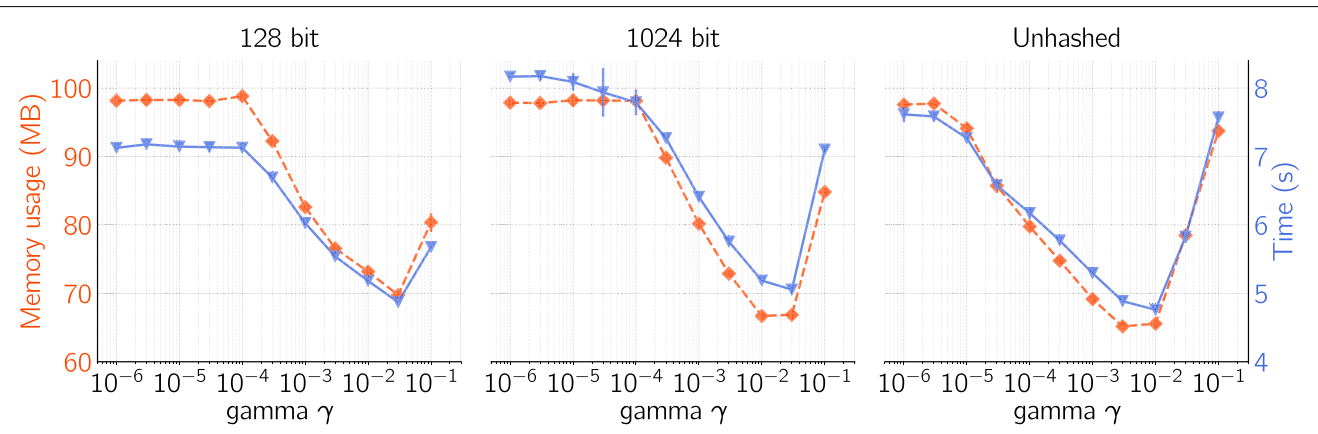

Fig. 4 Effect of gamma $(\gamma)$ and hash size on memory usage and run-time for Scikit SVM with Cost (C) equal to 1. Data points are average values of the four datasets, error bars are pooled standard deviations 


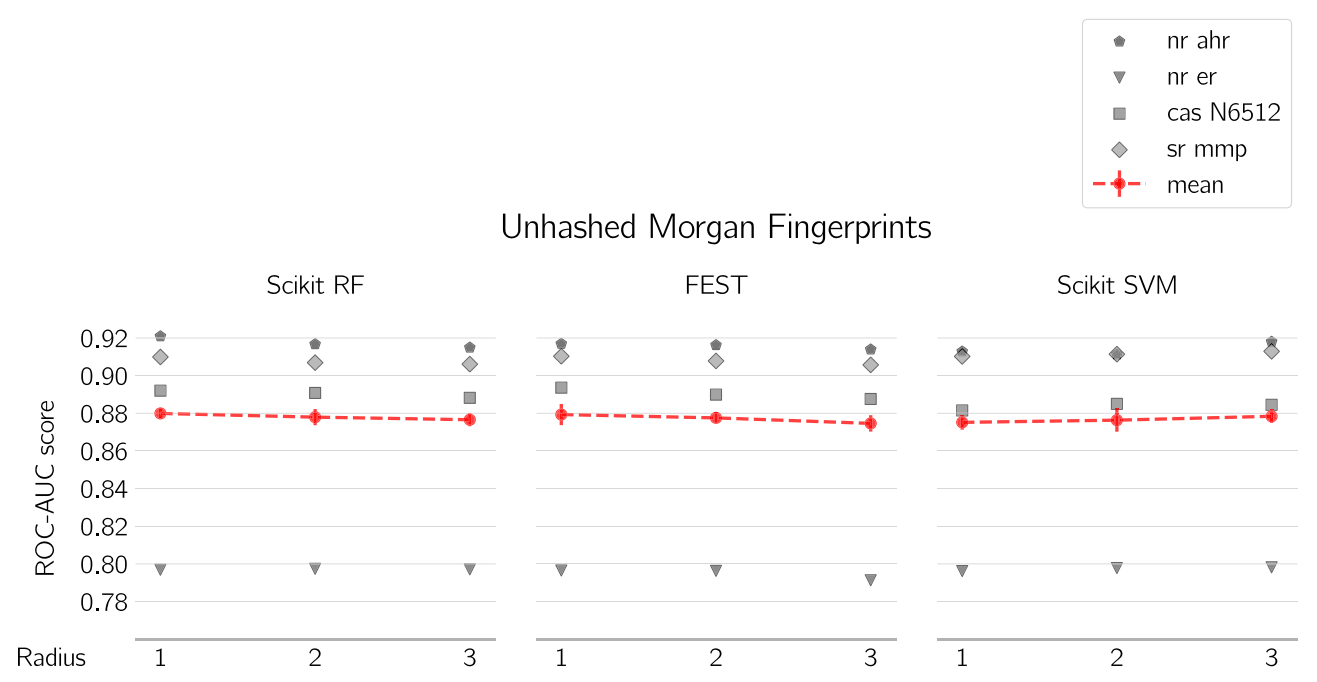

Signatures
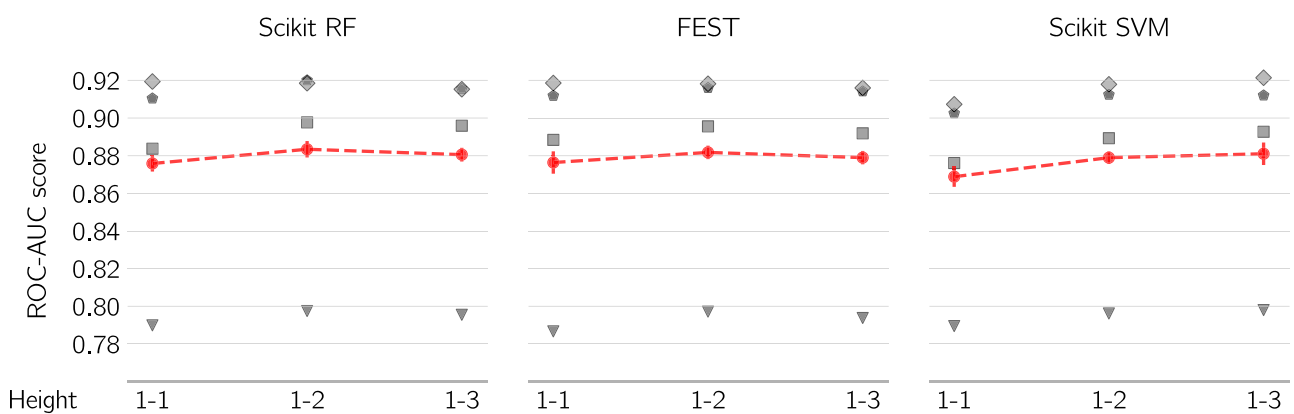

Fig. 5 Effect of fingerprint radii/height on predictive performance with Scikit RF, FEST and Scikit SVM. Grey markers represent the four datasets. Red markers/dashed lines indicate the average of the four datasets, with error bars being pooled standard deviations

unhashed fingerprints. This result is also in line with previously reported results on fingerprints in a virtual screening setting [39].

An advantage of using unhashed fingerprints is that the features have a particular substructure assigned to them and can therefore be traced back to the actual molecular features. Thus using unhashed fingerprints mean that each feature represents a certain molecular substructure, and by assessing feature importance, this can be helpful in interpreting model results in a chemical context [40-42].

An explanation of how memory usage and run-time for RF models trained on unhashed Morgan fingerprints are similar to hashed fingerprints could be that more information with unhashed fingerprints results in shorter trees, i.e. the splits are better. Concretely, hashed fingerprints are compressed and contain a lot of noise, which makes it harder for the trees to separate the class labels and reach leaf nodes.

\section{Effect of Max features for the two random forests}

In the case of Scikit RF, a clear decrease in memory usage can be observed with increasing number of features. This is highly correlated with the number of nodes in the trees (Fig. 3) indicating that more features to select from at each node results in better splits and shorter trees. Interestingly, this pattern cannot be observed with FEST. FEST however has a lower memory consumption and faster training than Scikit RF for all tested values of the Max features setting. Figure 4 illustrates the fast training-time of Scikit SVM, as well as its memory usage. Although the Scikit SVM could outperform RF in terms of run-time for a single run, Scikit SVM was sensitive to hyperparameter settings and required extensive grid-searches (see "Hyperparameter space of Scikit SVM" section).

\section{Effect of fingerprint radii}

We further investigated unhashed fingerprint radii including molecular signatures for comparison (Fig. 5). 
This is important in order to evaluate how increased sizes of substructures improve the separation of the two classes (i.e. improve predictive performance) by the ML methods.

For SVM it seems that more data does indeed results in a better model whereas for the random forest implementations no upward trend can be observed when increasing the radius (Fig. 5). One reason why no upward trend is seen with random forest could be that the number of features of radius/height 1 "drown" in the much larger number of features of radius/height 2 and 3 . The random subspace methods randomly selects a subset of features $m$ among the total number of features $M$, where $M$ is dominated by radii/heights 2 and 3 , which are more abundant than radii/heights of 1 and hence cannot reduce entropy to the same extent.

\section{Random forest and support vector machines}

As stated before it seems that SVM and RF perform very equally well on our data sets but it is interesting to note that as more data is included by adding more heights to the molecular signatures, or larger radii to the Morgan fingerprints, the RF seems to decrease in performance where SVM seems to increase. Based on this it seems reasonable to theorize that with more data and extensive grid search for the SVM parameters it is possible that SVM could perform better than RF but at much larger computational costs. If and when it is worth it probably varies from project to project. Also, we did not see this in our case, our Scikit SVM models did not perform better than our random forest models.

\section{Hyperparameter space of Scikit SVM}

For the comparison between RF and Scikit SVM, an extensive grid search for the hyperparameters of Scikit SVM was needed. We evaluated Cost (C) and gamma $(\gamma)$ according to Table 3 , by projecting the ROC-AUC scores of different hyperparameter combinations to a heat map (Fig. 6).

These heat plots illustrate the delicacy with which SVM models in QSAR (Fig. 6) must be treated where just a small space of possible hyperparameter values gives scores that compare with (and sometimes exceed) models from the random forest implementations. However, these observations agree with previous results from a study by Alvarsson et al. [43], where heat plots of models trained on molecular signatures were made with seven different public QSAR datasets. This suggest that the "hot spots" are found at very similar hyperparameter combinations for Morgan fingerprints and molecular signatures meaning that the parameter values that needs to be tested in order to optimise the SVM models actually are feasible and even though it requires more computation than random forest it could be justified in some cases.

\section{Availability}

All datasets and code for the analysis is available at: https ://github.com/pharmbio/kensert_rf_sparse, with an archived release at Zenodo http://doi.org/10.5281/zenod o.1291787.

\section{Conclusions}

We present evidence that hashing of Morgan fingerprints descriptors for QSAR modeling has a negative effect on predictive performance, with no significant improvement
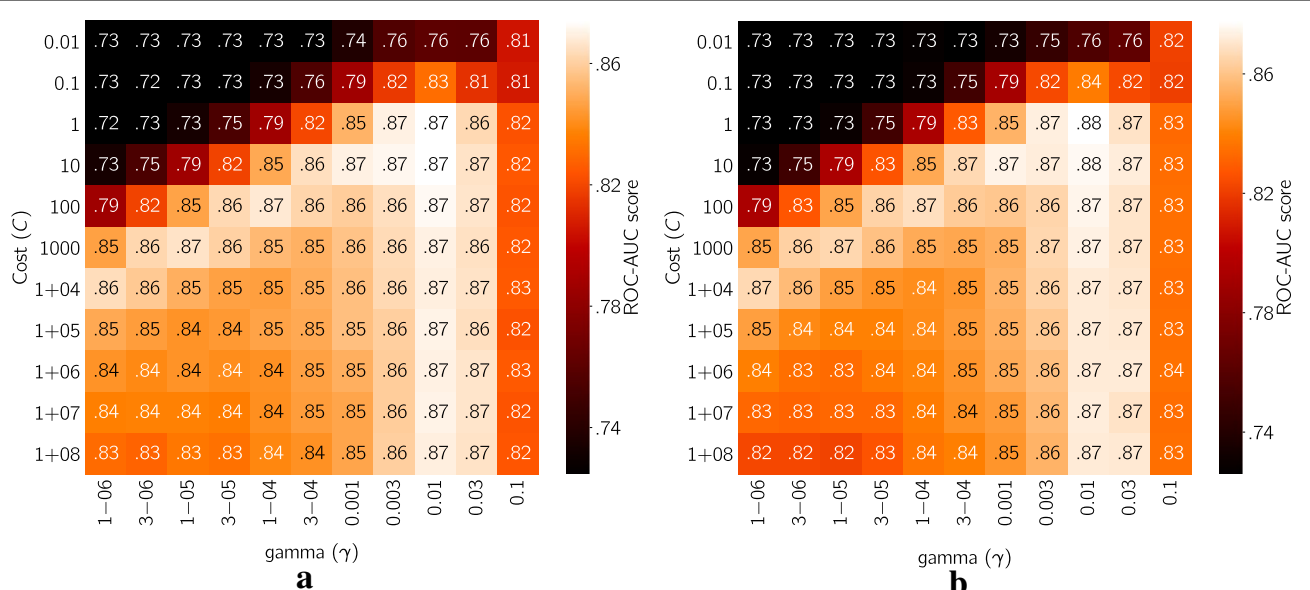

Fig. 6 Heat plots illustrating ROC-AUC scores with different hyperparameter combinations of Scikit SVM. a modelled on unhashed Morgan fingerprints with radius 3 ; $\mathbf{b}$ modelled on molecular signatures with height 1-3. Both plots are averages of the four datasets 
in computational efficiency. The FEST implementation was found to be capable of producing models of the same prediction quality as Scikit RF (SciKit), using less computational time and with lower memory requirements, however Scikit RF can be more easily parallelized on multi-core computers. The usefullness of this depends on the problem; building multiple smaller models is "embarrassingly parallelizable" and then the faster FEST implementation can be recommended but when building fewer large models than the built in parallelisation of Scikit RF will be relevant. For the Scikit implementation of random forest it was found that higher values for the Max features setting actually resulted in lower memory use but this could not be seen for FEST. Furthermore, no clear trend was identified that an increased number of features, i.e. increased radii/height, impacts favorably on the predictive performance for the random forest implementations. Evaluations of Scikit SVM and random forests demonstrate that both methods perform well but that SVM requires a more extensive grid search and tuning to reach high ROC-AUC scores but perhaps is better at taking advantage of the additional data found in higher radii of molecular signatures/Morgan fingerprints. Considering the easy and robust implementation of random forests this method could be considered a good initial choice for most cases and when the best results are needed SVM can be tested as well but at possibly a higher computational cost.

\section{Additional file}

Additional file 1: Table S1. Computed $p$ values according to the difference between the areas under two ROC curves using the method of Hanley and McNeil.

\section{Authors' contributions}

OS and UN conceived the project. AK carried out experiments and analysis. All authors were involved in study design, interpretation, and manuscript preparation. All authors read and approved the final manuscript.

\section{Author details}

${ }^{1}$ Department of Pharmaceutical Biosciences, Uppsala University, Uppsala, Sweden. ${ }^{2}$ Unit of Toxicology Sciences, Karolinska Institutet, Swetox, Forskarga$\tan 20$, SE-15136 Södertälje, Sweden. ${ }^{3}$ Department of Computer and Systems Sciences, Stockholm University, Box 7003, SE-164 07 Kista, Sweden.

\section{Acknowledgements}

The research at Swetox (UN) was supported by Knut and Alice Wallenberg Foundation and Swedish Research Council FORMAS. The computations were performed on resources provided by SNIC through Uppsala Multidisciplinary Center for Advanced Computational Science (UPPMAX) under Project SNIC 2017/7-241.

\section{Competing interests}

The authors declare that they have no competing interests.

\section{Appendix}

See Table 5.
Table 5 Effect of hash size on predictive performance with Scikit RF, Fest and Scikit SVM

\begin{tabular}{|c|c|c|c|c|}
\hline Method & Radius & Hash size & $\begin{array}{l}\text { Mean } \\
\text { ROC AUC }\end{array}$ & SD \\
\hline Scikit RF & 1 & 128 & 0.849175 & 0.0023297 \\
\hline Scikit RF & 1 & 256 & 0.854675 & 0.00229456 \\
\hline Scikit RF & 1 & 512 & 0.857525 & 0.00262345 \\
\hline Scikit RF & 1 & 1024 & 0.85965 & 0.00294236 \\
\hline Scikit RF & 1 & 2096 & 0.85945 & 0.00357561 \\
\hline Scikit RF & 1 & 4096 & 0.85945 & 0.00348676 \\
\hline Scikit RF & 1 & Unhashed & 0.879925 & 0.00144135 \\
\hline Scikit RF & 2 & 128 & 0.843675 & 0.00222542 \\
\hline Scikit RF & 2 & 256 & 0.853675 & 0.0033908 \\
\hline Scikit RF & 2 & 512 & 0.859175 & 0.00176494 \\
\hline Scikit RF & 2 & 1024 & 0.863025 & 0.0014151 \\
\hline Scikit RF & 2 & 2096 & 0.8633 & 0.00241454 \\
\hline Scikit RF & 2 & 4096 & 0.8644 & 0.00129808 \\
\hline Scikit RF & 2 & Unhashed & 0.87795 & 0.00214126 \\
\hline Scikit RF & 3 & 128 & 0.834875 & 0.00240936 \\
\hline Scikit RF & 3 & 256 & 0.8472 & 0.00245917 \\
\hline Scikit RF & 3 & 512 & 0.85435 & 0.00269165 \\
\hline Scikit RF & 3 & 1024 & 0.858525 & 0.00269258 \\
\hline Scikit RF & 3 & 2096 & 0.8603 & 0.0031249 \\
\hline Scikit RF & 3 & 4096 & 0.862875 & 0.00200624 \\
\hline Scikit RF & 3 & Unhashed & 0.876575 & 0.00171391 \\
\hline FEST & 1 & 128 & 0.849275 & 0.00152151 \\
\hline FEST & 1 & 256 & 0.856275 & 0.00226661 \\
\hline FEST & 1 & 512 & 0.85865 & 0.00271616 \\
\hline FEST & 1 & 1024 & 0.8594 & 0.00257633 \\
\hline FEST & 1 & 2096 & 0.860975 & 0.00175784 \\
\hline FEST & 1 & 4096 & 0.861525 & 0.00239008 \\
\hline FEST & 1 & Unhashed & 0.879325 & 0.00281158 \\
\hline FEST & 2 & 128 & 0.844275 & 0.00282975 \\
\hline FEST & 2 & 256 & 0.8531 & 0.00292104 \\
\hline FEST & 2 & 512 & 0.85865 & 0.00290086 \\
\hline FEST & 2 & 1024 & 0.8639 & 0.00189143 \\
\hline FEST & 2 & 2096 & 0.864225 & 0.00191703 \\
\hline FEST & 2 & 4096 & 0.86575 & 0.00242178 \\
\hline FEST & 2 & Unhashed & 0.87755 & 0.00140624 \\
\hline FEST & 3 & 128 & 0.83445 & 0.00234254 \\
\hline FEST & 3 & 256 & 0.84695 & 0.00355633 \\
\hline FEST & 3 & 512 & 0.8536 & 0.00216102 \\
\hline FEST & 3 & 1024 & 0.858325 & 0.00195704 \\
\hline FEST & 3 & 2096 & 0.86105 & 0.00356686 \\
\hline FEST & 3 & 4096 & 0.863875 & 0.00230326 \\
\hline FEST & 3 & Unhashed & 0.874625 & 0.00217658 \\
\hline Scikit SVM & 1 & 128 & 0.845025 & 0.00236326 \\
\hline Scikit SVM & 1 & 256 & 0.854125 & 0.00246475 \\
\hline Scikit SVM & 1 & 512 & 0.85575 & 0.00195512 \\
\hline Scikit SVM & 1 & 1024 & 0.857875 & 0.0023516 \\
\hline Scikit SVM & 1 & 2096 & 0.858425 & 0.00126293 \\
\hline Scikit SVM & 1 & 4096 & 0.85875 & 0.00224666 \\
\hline
\end{tabular}


Table 5 (continued)

\begin{tabular}{lllll}
\hline Method & Radius & Hash size & $\begin{array}{l}\text { Mean } \\
\text { ROC AUC }\end{array}$ & SD \\
\hline Scikit SVM & 1 & Unhashed & 0.875175 & 0.00193197 \\
Scikit SVM & 2 & 128 & 0.83875 & 0.00233024 \\
Scikit SVM & 2 & 256 & 0.848125 & 0.00232433 \\
Scikit SVM & 2 & 512 & 0.85155 & 0.00185876 \\
Scikit SVM & 2 & 1024 & 0.856875 & 0.00197927 \\
Scikit SVM & 2 & 2096 & 0.860825 & 0.00186615 \\
Scikit SVM & 2 & 4096 & 0.862725 & 0.00172699 \\
Scikit SVM & 2 & Unhashed & 0.87635 & 0.00306716 \\
Scikit SVM & 3 & 128 & 0.8283 & 0.00287359 \\
Scikit SVM & 3 & 256 & 0.838625 & 0.00278298 \\
Scikit SVM & 3 & 512 & 0.843375 & 0.00289093 \\
Scikit SVM & 3 & 1024 & 0.849425 & 0.00141067 \\
Scikit SVM & 3 & 2096 & 0.85605 & 0.00269629 \\
Scikit SVM & 3 & 4096 & 0.858475 & 0.00194936 \\
Scikit SVM & 3 & Unhashed & 0.8784 & 0.0017713 \\
\hline
\end{tabular}

Each data point is a mean of the four data sets. These values are plotted as Fig. 2 in the publication

\section{Publisher's Note}

Springer Nature remains neutral with regard to jurisdictional claims in published maps and institutional affiliations.

Received: 17 May 2018 Accepted: 3 October 2018 Published online: 11 October 2018

\section{References}

1. Tian S, LiY, Wang J, Zhang J, Hou T (2011) ADME evaluation in drug discovery. 9. Prediction of oral bioavailability in humans based on molecular properties and structural fingerprints. Mol Pharm 8(3):841-851. https:// doi.org/10.1021/mp100444g

2. Wu J, Zhang Q, Wu W, Pang T, Hu H, Chan WKB (2018) WDL-RF: predicting bioactivities of ligand molecules acting with $\mathrm{G}$ protein-coupled receptors by combining weighted deep learning and random forest. Bioinformatics 34:2271-2282. https://doi.org/10.1093/bioinformatics/bty070

3. Zhang H, Chen QY, Xiang ML, Ma CY, Huang Q, Yang SY (2009) In silico prediction of mitochondrial toxicity by using GA-CG-SVM approach. Toxicol in Vitro 23(1):134-140

4. Myshkin E, Brennan R, Khasanova T, SitnikT, Serebriyskaya T, Litvinova E (2012) Prediction of organ toxicity endpoints by QSAR modeling based on precise chemical-histopathology annotations. Chem Biol Drug Des 80:406-416

5. Low Y, Uehara T, Minowa Y, Yamada H, Ohno Y, Urushidani T (2011) Predicting drug-induced hepatotoxicity using QSAR and toxicogenomics approaches. Chem Res Toxicol 24(8):1251-1262. https://doi.org/10.1021/ tx200148a

6. Polishchuk PG, Muratov EN, Artemenko AG, Kolumbin OG, Muratov NN, Kuz'min VE (2009) Application of random forest approach to QSAR prediction of aquatic toxicity. J Chem Inf Model 49(11):2481-2488. https ://doi.org/10.1021/ci900203n

7. Rogers D, Hahn M (2010) Extended-connectivity fingerprints. J Chem Inf Model 50(5):742-754

8. Jensen BF, Vind C, Brockhoff PB, Refsgaard HHF (2007) In silico prediction of cytochrome P450 2D6 and 3A4 inhibition using Gaussian kernel weighted k-nearest neighbor and extended connectivity fingerprints, including structural fragment analysis of inhibitors versus noninhibitors. J Med Chem 50(3):501-511. https://doi.org/10.1021/jm060333s

9. Rogers D, Brown RD, Hahn M (2005) Using extended-connectivity fingerprints with Laplacian-modified Bayesian analysis in high-throughput screening follow-up. J Biomol Screen 10(7):682-686. https://doi. org/10.1177/1087057105281365

10. Zhou D, Alelyunas Y, Liu R (2008) Scores of extended connectivity fingerprint as descriptors in QSPR study of melting point and aqueous solubility. J Chem Inf Model 48(5):981-987. https://doi.org/10.1021/ci800 $024 c$

11. Yao XJ, Panaye A, Doucet JP, Zhang RS, Chen HF, Liu MC (2004) Comparative study of QSAR/QSPR correlations using support vector machines, radial basis function neural networks, and multiple linear regression. J Chem Inf Comput Sci 44(4):1257-1266. https://doi.org/10.1021/ci049965i

12. Cortes-Ciriano I (2016) Benchmarking the predictive power of ligand efficiency indices in QSAR. J Chem Inf Model 56(8):1576-1587. https:// doi.org/10.1021/acs.jcim.6b00136

13. Norinder U (2003) Support vector machine models in drug design: applications to drug transport processes and QSAR using simplex optimisations and variable selection. Neurocomputing 55(1):337-346

14. Zhou XB, Han WJ, Chen J, Lu XQ (2011) QSAR study on the interactions between antibiotic compounds and DNA by a hybrid genetic-based support vector machine. Monatshefte fuer Chemie/Chemical Monthly 142(9):949-959. https://doi.org/10.1007/s00706-011-0493-7

15. Breiman $L$ (2001) Random forests. Mach Learn 45(1):5-32

16. Carlsson L, Helgee EA, Boyer S (2009) Interpretation of nonlinear QSAR models applied to Ames mutagenicity data. J Chem Inf Model 49(11):2551-2558. https://doi.org/10.1021/ci9002206

17. Cannon EO, Bender A, Palmer DS, Mitchell JBO (2006) Chemoinformaticsbased classification of prohibited substances employed for doping in sport. J Chem Inf Model 46(6):2369-2380. https://doi.org/10.1021/ci060 1160

18. Henriksson A, Zhao J, Dalianis H, Boström H (2016) Ensembles of randomized trees using diverse distributed representations of clinical events. BMC Med Inf Decis Mak 16(2):69. https://doi.org/10.1186/s1291 1-016-0309-0

19. Karlsson I, Boström H (2014) Handling sparsity with random forests when predicting adverse drug events from electronic health records. In: 2014 ieee international conference on healthcare informatics, 15-17 September 2014, Verona. IEEE, pp 17-22

20. Svetnik V, Liaw A, Tong C, Wang T (2004) Multiple classifier systems. In: Proceedings. Springer, Berlin

21. Svetnik V, Liaw A, Tong C, Culberson JC, Sheridan RP, Feuston BP (2003) Random forest: a classification and regression tool for compound classification and QSAR modeling. J Chem Inf Comput Sci 43(6):1947-1958. https://doi.org/10.1021/ci034160g

22. Morgan $\mathrm{HL}$ (1965) The generation of a unique machine description for chemical structures: a technique developed at chemical abstracts service. J Chem Doc 5(2):107-113. https://doi.org/10.1021/c160017a018

23. Norinder U, Ek ME (2013) QSAR investigation of NaV1.7 active compounds using the SVM/signature approach and the bioclipse modeling platform. Bioorg Med Chem Lett 23(1):261-263

24. Chen JJF, Visco DP Jr (2017) Developing an in silico pipeline for faster drug candidate discovery: virtual high throughput screening with the signature molecular descriptor using support vector machine models. Chem Eng Sci 159:31-42

25. Huang R, Xia M, Nguyen D-T, Zhao T, Sakamuru S, Zhao J, Shahane SA Rossoshek A, Simeonov A (2016) Tox21 challenge to build predictive models of nuclear receptor and stress response pathways as mediated by exposure to environmental chemicals and drugs. Front Environ Sci 3:85

26. Hansen K, Mika S, Schroeter T, Sutter A, ter Laak A, Steger-Hartmann T (2009) Benchmark data set for in silico prediction of Ames mutagenicity. J Chem Inf Model 49(9):2077-2081

27. Hunter JD (2007) Matplotlib: a 2D graphics environment. Comput Sci Eng 9(3):90-95

28. Waskom M, Botvinnik O, O'Kane D, Hobson P, Lukauskas S, Gemperline DC et al (2017) mwaskom/seaborn: v0.8.1. https://doi.org/10.5281/zenod 0.54844

29. Landrum G (2017) RDKit documentation 2017.09.01 release. http://www. rdkit.org/RDKit_Docs.current.pdf. Accessed 15 Nov 2017 
30. CPSign (2008). http://cpsign-docs.genettasoft.com. Accessed 04 June 2018

31. Breiman L, Friedman J, Stone CJ, Olshen RA (1984) Classification and regression trees. Chapman and Hall, London

32. Bernard S, Heutte L, Adam S (2010) A study of strength and correlation in random forests. In: Huang DS, McGinnity M, Heutte L, Zhang XP (eds) Advanced intelligent computing theories and applications. Springer, Berlin, pp 186-191

33. Raileanu LE, Stoffel K (2004) Theoretical comparison between the Gin index and information gain criteria. Ann Math Artif Intell 41(1):77-93. https://doi.org/10.1023/B:AMAl.0000018580.96245.c6

34. Karampatziakis $N$ (2008) Fast ensembles of sparse trees. http://lowra nk.net/nikos/fest/. Accessed 15 Nov 2017

35. Pedregosa F, Varoquaux G, Gramfort A, Michel V, Thirion B, Grisel O (2011) Scikit-learn: machine learning in Python. J Mach Learn Res 12:2825-2830

36. Chang CC, Lin CJ (2011) LIBSVM: a library for support vector machines. ACM Trans Intell Syst Technol 2(3):27

37. Bradley AP (1997) The use of the area under the ROC curve in the evaluation of machine learning algorithms. Pattern Recognit 30(7):1145-1159
38. Hanley JA, McNeil BJ (1982) The meaning and use of the area under a receiver operating characteristic (ROC) curve. Radiology 143(1):29-36. https://doi.org/10.1148/radiology.143.1.7063747

39. Sastry M, Lowrie JF, Dixon SL, Sherman W (2010) Large-scale systematic analysis of 2D fingerprint methods and parameters to improve virtual screening enrichments. J Chem Inf Model 50(5):771-84

40. Spjuth O, Eklund M, Ahlberg Helgee E, Boyer S, Carlsson L (2011) Integrated decision support for assessing chemical liabilities. J Chem Inf Model 51(8):1840-7

41. Ahlberg E, Spjuth O, Hasselgren C, Carlsson L (2015) Interpretation of conformal prediction classification models. In: International symposium on statistical learning and data sciences. Springer, Berlin, pp 323-334

42. Lapins M, Arvidsson S, Lampa S, Berg A, Schaal W, Alvarsson J (2018) A confidence predictor for logD using conformal regression and a supportvector machine. J Cheminform 10(1):17

43. Alvarsson J, Eklund M, Andersson C, Carlsson L, Spjuth O, Wikberg JES (2014) Benchmarking study of parameter variation when using signature fingerprints together with support vector machines. J Chem Inf Model 54(11):3211-3217. https://doi.org/10.1021/ci500344v
Ready to submit your research? Choose BMC and benefit from:

- fast, convenient online submission

- thorough peer review by experienced researchers in your field

- rapid publication on acceptance

- support for research data, including large and complex data types

- gold Open Access which fosters wider collaboration and increased citations

- maximum visibility for your research: over 100M website views per year

At BMC, research is always in progress.

Learn more biomedcentral.com/submissions 